\title{
Treatment of advanced neuroendocrine tumours with the radiolabelled somatostatin analogue octreotide
}

\author{
Gregory A. Kaltsas ${ }^{1}$, Zoe Stefanidou ${ }^{2}$, Dimitris Papadogias ${ }^{1}$, Ashley B. Grossman ${ }^{3}$ \\ Department of Endocrinology, ${ }^{1}$ George Genimatas General Hospital, Athens, Greece, ${ }^{2}$ Novartis Hellas, \\ ${ }^{3}$ St Bartholomew's Hospital, ECIA 7BE, London, UK
}

\begin{abstract}
Neuroendocrine tumours (NET) have a particular tendency to express functional receptors and/or uptake mechanisms. Radionuclides, such as ${ }^{111}$ In-pentetreotide, a somatostatin analogue, which bind to somatostatin receptors, present an imaging modality that has been used for both the diagnosis and staging of NET. Scintigraphy with ${ }^{111}$ In-pentetreotide can identify lesions beyond the diagnostic sensitivity of conventional imaging modalities. In addition, NET that demonstrate positive uptake to a diagnostic ${ }^{111}$ In-pentetreotide can, in theory, be treated with these radionuclides, thus presenting a novel and evolving therapeutic modality in addition to other traditional therapeutic approaches. Although experience with ${ }^{90}$ Y-DOTA-D-Phe ${ }^{1}-$ Tyr $^{3}$-octreotide therapy is still limited, preliminary studies have demonstrated useful activity in a variety of NET with limited side-effects. Large phase II clinical trials using ${ }^{90}$ Y-DOTA-D-Phe ${ }^{1}-$ Tyr $^{3}$-octreotide therapy are still on-going and the results are expected to be presented soon. In order to improve the response rates obtained, newer somatostatin analogues are being developed and the combination with other $\beta$-emitting particles in addition to ${ }^{90} \mathrm{Y}$ is being considered.
\end{abstract}

Key words: neuroendocrine, tumours, radionuclides

\section{INTRODUCTION}

Neuroendocrine tumours (NET) constitute a heterogeneous group of tumours which originate from the diffuse neuroendocrine system (DNES) and are programmed to adopt a specific neuroendocrine phenotype ${ }^{1}$. The system includes endocrine glands, such as the pituitary, the parathyroids and adrenal glands

Address correspondence and requests for reprints to: Dr. Gregory Kaltsas, George Genimatas General Hospital, Athens, Greece, Tel: 003010 7796043, Fax: 003010 7779146, E-mail: gakaltsas@mds.qmw.ac.uk

Received 14-03-02, Revised 29-04-02, Accepted 30-05-02 as well as endocrine islets within glandular tissue (thyroid or pancreatic) and cells disseminated between exocrine cells, such as endocrine cells of the digestive and respiratory tracts ${ }^{2,3}$. Although NET may vary in clinical presentation, location and specific histology, they share common features which are detected by refined morphological techniques for endocrine granules, histochemical techniques for secretory peptides or amines and electron microscopy ${ }^{3}$. Some NET may occasionally exert a very aggressive behavior and become highly malignant (poorly-differentiated NET), but the great majority tend to be relatively slow growing (well-differentiated NET) and retain many multipotent differentiation capacities ${ }^{1,3}$. Such features 
include the possession of neuroamine uptake mechanisms and/or specific receptors at the cell membrane, such as somatostatin receptors, which can be of great value in identifying and localizing these tumours as well as in their therapy.

\section{${ }^{111}$ IN-PENTETREOTIDE IN THE DIAGNOSIS OF NEUROENDOCRINE TUMOURS}

Radionuclides represent a diagnostic modality where radiolabelled peptide analogues, based on their ability to bind to suitable ligands, have been utilized for the identification and localization of $\mathrm{NET}^{4}$. The use of radionuclides presents an imaging modality based on physiological characteristics (the presence of functioning receptors and uptake mechanisms) rather than on purely anatomical alterations. This imaging modality has been extensively used for the diagnosis and treatment of NET as the great majority of them express somatostatin receptors and therefore become an ideal target for somatostatin derived radionuclides ${ }^{4,5}$.

Somatostatin is a 14 aminoacids-peptide hormone that is present in the hypothalamus, the cerebral cortex, the brainstem, the gastrointestinal system and the pancreas, whereas somatostatin receptors have been identified on many cells of neuroendocrine origin ${ }^{6,7}$. Somatostatin is mainly an inhibitory peptide that has a very short half-life, approximately 2 minutes, which makes it unsuitable for routine clinical use ${ }^{7,8}$. Introduction of D-amino acids and shortening of the molecule to the bioactive core sequence resulted in the eight amino acids-containing somatostatin analogues ${ }^{1,6,7}$. Octreotide (Novartis, Basel, Switzerland) is the first somatostatin analogue to be used in clinical practice while considerable experience has also been obtained with lanreotide (Ipsen, Paris, France) and more recently vapreotide (Figure 1$)^{9}$. These compounds can be conjugated with DOTA $(1,4,7,10$ tetraazacyclododecane-1,4,7,10-tetraacetic acid) as a way of coupling somatostatin analogues with various radionuclides ${ }^{5,8}$. Out of the five subtypes of somatostatin receptors that have been described, somatostatin receptor type 2 is the one most frequently found in $\mathrm{NET}^{5,8}$. Octreotide exerts its highest affinity for somatostatin receptors type 2, 3 and 5 and thus scintigraphy with octreotide reflects the visualization of octreotide-binding somatostatin receptors ${ }^{7,8}$. In general, "well-differentiated" NET usually express soma-
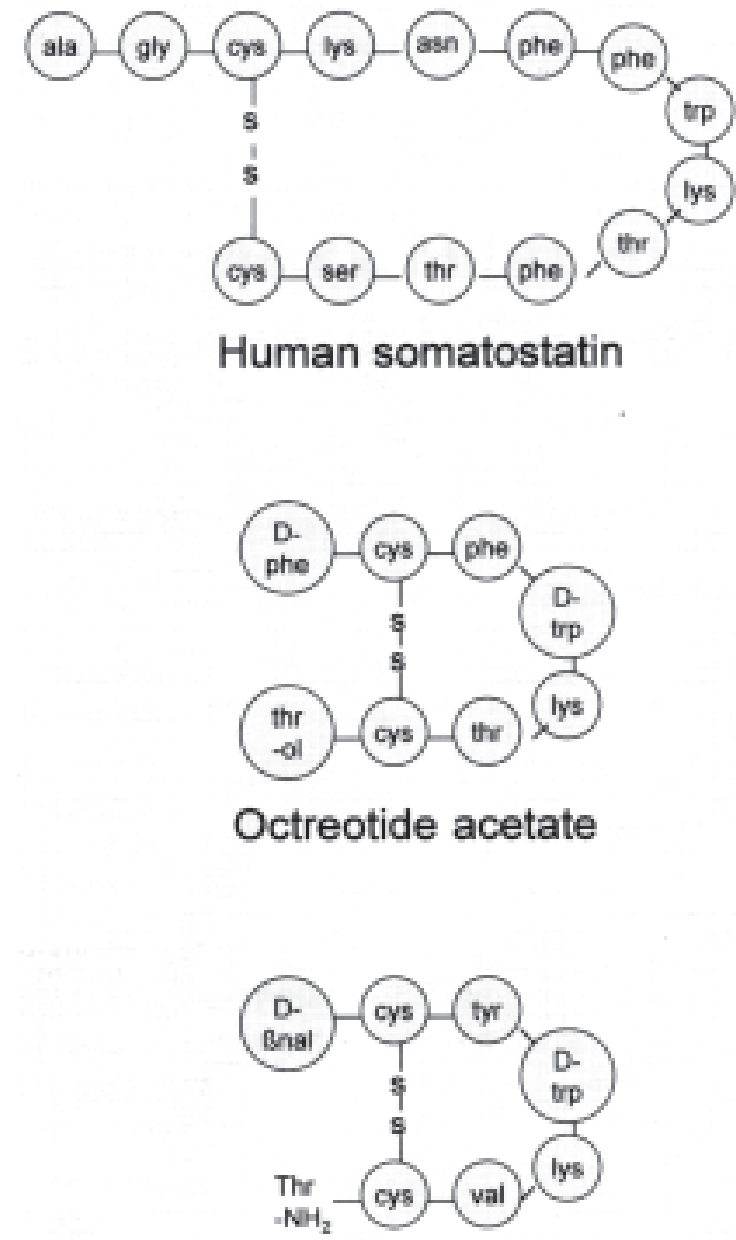

lanreotide

Figure 1. Structure of human somatostatin - 14 and its long acting analogues which are currently used in clinical practice

tostatin receptors which are generally absent from "poorly-differentiated" tumours ${ }^{2,9}$.

Covalently linking ethilene-triamine-penta-acetic acid (DTPA) with octreotide results in DTPA-octreotide (pentetreotide); this tracer was initially labelled with ${ }^{123} \mathrm{I}$, but is more clinically effective with ${ }^{111} \mathrm{In}^{10}$. Scintigraphy with ${ }^{111}$ In-pentetreotide has been shown to have a detection rate of $67-91 \%$ for all NET but is of low specificity as uptake is also demonstrable in many other tumours, granulomas and autoimmune diseases $^{4,7,11}$. (Table 1 shows the particular sensitivity of ${ }^{111}$ In-pentetreotide in the identification and localization of various NET). The fact that abdominal single photon emission computed tomography (SPECT) was not routinely performed in some of the original 
Table 1. ${ }^{111}$ In-pentetreotide imaging results in a variety of neuroendocrine tumours

\begin{tabular}{lc}
\hline Tumour & $\begin{array}{c}\text { Percent tumours with } \\
\text { 111 In-pentetreotide uptake }\end{array}$ \\
\hline Carcinoid & $96 \%$ \\
Pheochromocytoma & $86 \%$ \\
Medullary thyroid carcinoma & $71 \%$ \\
Paraganglioma & $94 \%$ \\
Insulinoma & $61 \%$ \\
Glucagonoma & $100 \%$ \\
Gastrinoma & $100 \%$ \\
\hline
\end{tabular}

Adopted from Wiseman and Kvols ${ }^{11}$

studies may explain the differences in the detection ability reported between several studies ${ }^{4,8,11,12}$. Scintigraphy with ${ }^{111}$ In-pentetreotide is used for the diagnosis and staging of the tumours as well as identifying the patients who may be suitable for treatment with radiolabelled octreotide ${ }^{1,4,7}$. The detection of an unsuspected lesion in a patient with known metastatic spread usually has little impact on management. In contrast, the detection of such a lesion in patients with a single known lesion or without any known lesion is important in that it may affect the selection of curative surgery, which remains the treatment of choice in patients with $\mathrm{NET}^{8}$. However, there are no clinical or biochemical predictors of a positive scan, and there is no correlation of a positive scan with the anatomical site of the origin of the tumour ${ }^{4,12}$. In spite of the efforts in developing more specific radioligands, at present ${ }^{111}$ In-pentetreotide remains the radiopharmaceutical of first choice for the imaging of NET ${ }^{10}$. The injected activity in adults is $110-220 \mathrm{MBq}$ of ${ }^{111}$ In-pentetreotide; approximately $80 \%$ of i.v. administered radiolabelled ${ }^{111} \mathrm{In}$-pentetreotide is eliminated via the urinary system ${ }^{10}$.

\section{BASIC CONCEPTS OF APPLYING RADIOPHARMACEUTICAL THERAPY IN NEUROENDOCRINE TUMOURS}

Many tumours are highly radiosensitive, but delivering an adequate dose to the tumour can result in damage to the surrounding healthy tissue. Coupling a radioisotope to a molecule which would specifically bind to tumour cells could, in theory, deliver an effective radiation dose to the tumour without damage to non-tumour tissues, potentially limiting adverse effects $^{8,11,12}$. The success of the therapeutic strategy depends upon the amount of radioligand which can be concentrated within tumour cells and the rates of internalization, degradation and recycling of both ligand and receptor ${ }^{8,11}$. Targeted radiotherapy will be effective in tumour treatment if all or nearly all tumour cells have the target antigen, receptor or chemical structure for the radioisotope for the carrier molecule to bind ${ }^{11,13}$. Tumour heterogeneity will cause incomplete responses at best unless the radiation delivered can kill the nearby tumor cells that are targetnegative $^{8,11}$. Thus, the radioisotope physical properties are important for targeting radiation to the target positive tumour cells but also for the target negative cells as their death will depend on the crossfire from the radioisotope localized on or in the targetpositive tumour cells ${ }^{11,13}$. This non-targeted radiation will also contribute to the radiation dose being absorbed by the surrounding normal cells ${ }^{13}$. The selection of the best radioisotope to use for target radiotherapy depends on the type of radiation emitted, the emission energies, the distance over which the energy is deposited and the physical half-life of the radioisotope (Table 2$)^{8,11}$.

Radioiodine for treatment of thyroid disorders has been the best example of a radionuclide targeting

Table 2. $\beta$-Emitting Radionuclides for Targeted Radiotherapy

\begin{tabular}{cccc}
\hline Radionuclide & Gamma Emission $(\mathbf{k e v})$ & Physical Half-Life $\left(\mathbf{T}_{12}\right.$, days $)$ & Beta Emission X90 $(\mathbf{m m}$ in water $)$ \\
\hline${ }^{153} \mathrm{Sm}$ & 103 & 1.93 & 1.01 \\
${ }^{90} \mathrm{Y}$ & - & 2.67 & 5.34 \\
${ }^{186} \mathrm{Re}$ & 137 & 3.78 & 1.8 \\
${ }^{17} \mathrm{Lu}$ & 113,208 & 6.68 & - \\
${ }^{131} \mathrm{I}$ & 365,637 & 8.04 & 0.83 \\
${ }^{32} \mathrm{P}$ & - & 14.28 & 3.61 \\
${ }^{89} \mathrm{Sr}$ & - & 50.52 & \\
\hline
\end{tabular}

Adopted from Wiseman and Kvols ${ }^{11}$ 
agent with a high target-to-nontarget ratio, rapid clearance of the unbound radioisotope and a long residence time in the target ${ }^{11}$. With the introduction of ${ }^{131} \mathrm{I}$-labelled metaiodobenzylguanidine (MIBG), the field of radionuclide treatment has been extended to a wide range of $\mathrm{NET}^{11,12,14}$. Metaiodoenzylguanidine is taken up actively by cell membranes and then by neurosecretory granules in the cytoplasm of cells that express amino-uptake mechanisms; more than $90 \%$ of pheochromocytomas and paragangliomas, $70 \%$ of carcinoids and around $35 \%$ of medullary thyroid carcinomas concentrate $\mathrm{MIBG}^{2,12,15}$. Based on a positive scintigraphy with ${ }^{123} \mathrm{I}-\mathrm{MIBG}$, treatment with ${ }^{131} \mathrm{I}$-MIBG has produced overall objective tumor responses ranging between $7.5-35 \%$ in a variety of NET (higher response rates have been obtained in patients with chromaffin cell tumours) ${ }^{2,12,15}$. As scintigraphy with ${ }^{111}$ In-pentetreotide is more sensitive than scintigraphy with ${ }^{123} \mathrm{I}$ MIBG in identifying various types of $\mathrm{NET}^{4,12}$, it is expected that treatment with radiolabelled somatostatin analogues will probably be applicable to a larger population of patients with NET as well as locate the same lesions identified by ${ }^{131} \mathrm{I}-\mathrm{MIBG}^{4,12}$.

\section{RADIOPHARMACEUTICAL THERAPY WITH ${ }^{111}$ IN OCTREOTIDE}

${ }^{111}$ In emits mainly $\gamma$-radiation, which passes through tissue relatively easily and can be imaged using a $\gamma$ scanner ${ }^{5}$. In addition, ${ }^{111}$ In also emits Auger and conversion electrons having particle ranges of 0.02 to 10 $\mu \mathrm{m}$ and 200 to $500 \mu \mathrm{m}$ respectively and can therefore be used to investigate its antiproliferative effect in malignant tumours ${ }^{8,11}$. The short ranged Auger electrons are expected to exert an effect on tumour cell proliferation as the radiotoxicity of Auger electrons is very high if their target, the DNA of the cell, is within the particle range ${ }^{8,16}$. Following initial experiments in rats where a tumouricidal effect of the radiopharmaceutical was established, the treatment was approved for phase I study in humans ${ }^{8,13}$. Following dosimetry and pharmacokinetic data derived from animal studies, ${ }^{111}$ In-octreotide was given in humans with at least 2-week intervals between administrations and a total of 8 administrations was aimed at $^{8}$. Potential hematological, biochemical and endocrine side-effects were examined and patients were scanned 3 and 7 days after each administration of the radiotherapeutical dose. The percentage uptake of the administered dose in total body and in the most prominent tumour was calculated $^{8}$. With administered doses of 6000 to 7000 $\mathrm{MBq}$, critical organs were shown to be the kidney and the spleen. Thirty end stage patients with NET were treated; twenty-one of these patients received a cumulative dose of $20 \mathrm{GBq}\left[{ }^{111}\right.$ InDTPA] octreotide ${ }^{8}$. Seven patients who were treated with a total dose of less than $20 \mathrm{GBq}$ had to stop therapy prematurely due to the extension of their disease despite treatment with ${ }^{111}$ In-DTPA-octreotide ${ }^{8}$. From the 21 patients who received adequate doses of ${ }^{111}$ In-DTPA-octreotide (all had progressive disease), 6 showed an antiproliferative effect demonstrated as shrinkage of the tumour. In addition, in another 8 patients treatment with ${ }^{111} \mathrm{In}$ DTPA-octreotide resulted in stabilization of their disease (Figure 2$)^{8}$. However, in spite of these moderately encouraging preliminary results, the radionuclides used in receptor scintigraphy, such as ${ }^{111} \mathrm{In}$, are in general not as suitable for radiotherapy as potent B-emitters, such as yttrium $90\left({ }^{90} \mathrm{Y}\right)^{8,11-13}$.

\section{RADIOPHARMACEUTICAL THERAPY WITH ${ }^{90}$ Y OCTREOTIDE}

Radionuclides emitting B-radiation have greater therapeutic potential since the particles they emit have sufficient energy to cause cell damage without penetrating very far into surrounding tissue $\mathrm{e}^{5,8,11}$. A highenergy B-emitter attached to a somatostatin receptor analogue could potentially deliver a lethal dose of radiation to a somatostatin receptor-positive tumour with minimal damage to healthy tissue. The same somatostatin analogue ( $\mathrm{Tyr}^{3}$-octreotide) used for diagnosis has been coupled to a bifunctional chelating agent (DOTA) for the complexing of ${ }^{90} \mathrm{Y}$, a pure energetic $\beta$-emitter well suited for internal radiotherapy ${ }^{2,8,11-13}$. In contrast to ${ }^{90} \mathrm{Y}$-DTPA-octreotide, ${ }^{90} \mathrm{Y}$ DOTA-D-Phe ${ }^{1}$-Tyr ${ }^{3}$-octreotide, $\left({ }^{90} \mathrm{Y}\right.$-SMT487, OctreoTher $^{T M}$ ), shows no dissociation from the DOTA chelated peptide in serum and can deliver tumouricidal doses of radiation by targeting somatostatin receptor positive tumours who have demonstrated uptake on a diagnostic ${ }^{111}$ In-pentetreotide scan (Octreoscan $)^{8,13}$. SMT487 binds with high affinity to somatostatin receptors (subtypes 2 and 3 ) and retains both its binding properties and physiological function when labelled with ${ }^{90} \mathrm{Y}^{17}$. OctreoTher induced significant tumour regression in nude mice bearing human pancreatic carcinoma cells and prolonged survival ${ }^{8,17}$. These 


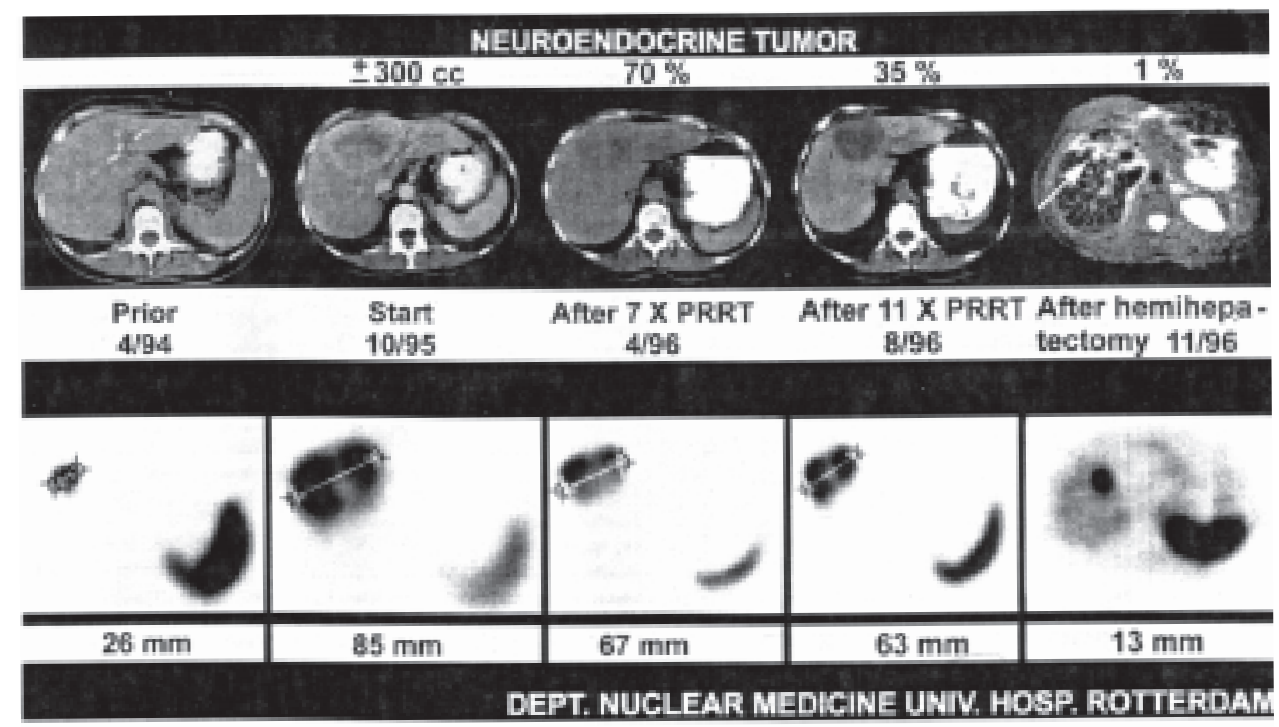

Figure 2. Effects of radiotherapy with [111 In-DTPA] octreotide in a patient with a hepatic metastasis of a neuroendocrine tumour treated according to the described protocol. The upper panel shows transversal CT scans, except on 11/96 (MRI), and the lower panel illustrates transversal slices of [ $\left.{ }^{111} \mathrm{In}-\mathrm{DTPA}\right]$ octreotide SPECT images. The decline in tumour size $(300 \mathrm{cc})$ was $30 \%$ after seven treatment courses and $65 \%$ after 11 courses After the effect of 11 treatment-courses, the option of hemi-hepatectomy became feasible and this procedure was carried out, leaving in situ a lesion of 9mm diameter (MRI) at the cutting-edge (Reproduced with permission from Ann Oncol, 10 (Suppl 2): S23-S29, 1999).

good preclinical results led to phase I clinical trials mainly with OctreoTher and various other ${ }^{90} \mathrm{Y}$-labelled somatostatin analogues ${ }^{17}$. Phase I clinical trials conducted in patients with NET established that after an intravenous infusion, plasma radioactivity disposition of OctreoTher was multiphasic (terminal t1/2 approximately $8.6 \mathrm{~h}$ ); unbound radioactivity was excreted in urine ${ }^{17}$. By $24 \mathrm{hrs}$, post-dose plasma radioactivity was $<1 \%$ of the injected dose while OctreoTher was not significantly taken up by erythrocytes. A correlation between creatinine clearance and total body radioactivity clearance was observed reflecting that the kidney is the principal drug elimination organ. It was therefore suggested that OctreoTher is not recommended in subjects whose creatinine clearance is less than $40 \mathrm{ml} / \mathrm{min}^{8,17}$. Radioactivity exposure was mainly, besides the kidneys, to the spleen, urinary bladder wall and tumours. The relative biodistribution and pharmacokinetic profile of OctreoTher was not altered by changes in the absolute peptide dose over the range of 50-500 mcg while concomitant administration of amino acids reduced renal radioactivity uptake without altering tumour uptake in a phase I study ${ }^{17}$. Cationic amino acids appear to be responsible for the "blocking" of renal tubule uptake of proteins or peptides $^{18}$. This is because the membranes of renal tu- bules contain negatively charged sites to which positively charged residues of peptides or proteins are thought to bind ${ }^{8,17,18}$. An inhibition of this binding site may explain the effects by administering the positively charged amino acid lysine (both D- and L-lysine) on the re-uptake of OctreoTher. A variety of other maneuvers have been attempted, so far without success, in further lowering the renal uptake of OctreoTher $^{8,17}$.

In another similar preliminary study of 20 patients with a variety of tumours, Paganelli et al. reported results for an escalating dosage using OctreoTher ${ }^{19}$. Treatment was initiated with $1.1 \mathrm{GBq}$ per cycle (3 cycles per patient), increasing by $0.4 \mathrm{GBq}$ increments. The maximum tolerated dose was not stated but dosimetric estimations showed that the highest doses were delivered to kidneys $(3.3 \pm 2.2 \mathrm{mGy} / \mathrm{MBq})$ and spleen $(7.6 \pm 6.3 \mathrm{MBq} / \mathrm{MBq})$. Only one patient showed grade II renal toxicity and five had hematological toxicity of the same grade. For higher activities renal or splenic toxicities could be prevented by intravenous administration of D-lysine: the objective response rate (complete + partial responses) was $20 \%{ }^{2,19}$. Phase I clinical trials results support the safety and tolerability of the dose selected for further study. A Novartis-sponsored 
phase II study involving centers in the USA, Europe and Australia is currently on-going to test the efficacy of OctreoTher in patients with a variety of disseminated NET and the results will soon be published ${ }^{10,17}$. While in general OctreoTher is a non- $\gamma$ emitter, the $\beta$-particles can cause localised release of $\gamma$-activity from affected cells ('bremsstrahlung'), and we have found that some imaging of the effectiveness of therapy is possible (Figure 3). A fixed-dose regimen of $120 \mathrm{mCi} /$ cycle x 3 cycles administered with concomitant amino acid infusion has been chosen for the study ${ }^{10,17}$. Until the results of this study are published, treatment with radiolabelled somatostatin analogues will remain an attractive alternative therapeutic option to current available therapeutic modalities.

Currently, there is very little experience in using ${ }^{90}$ Y-DOTATOC, a compound which appears to be identical to OctreoTher, for the treatment of metastatic NET compared to that of ${ }^{131} \mathrm{I}-\mathrm{MIBG}^{20,21} .{ }^{90} \mathrm{Y}$ DOTATOC is a peptide vector DOTA-D-Phe1-Tyr ${ }^{3}$ Octreotide (DOTATOC; DOTA: 1,4,7,10-tetra-azacyclododecane-N, N',N",N"'-tetraacetic acid), stably labelled with yttrium- $90^{20,21}$. Out of ten patients with somatostatin receptor-positive metastatic NET treated with ${ }^{90}$ Y-DOTATOC, the 6 patients who received multiple doses reported improvement in tumour growth, hormonal secretion and symptoms, with minimal side-effects, while the others showed stable disease, suggesting that ${ }^{90} \mathrm{Y}$-DOTATOC is a potentially valuable therapy for somatostatin receptor-positive tumours (Figure 4$)^{21}$. In total, 24 patients have been treated with ${ }^{90} \mathrm{Y}$-DOTATOC and received $<7,400$ $\mathrm{MBq} / \mathrm{m}^{2},{ }^{21}$. This dose corresponds to $200 \mathrm{mCi} / \mathrm{m}^{2}$, and some of the administered cycles were in the presence

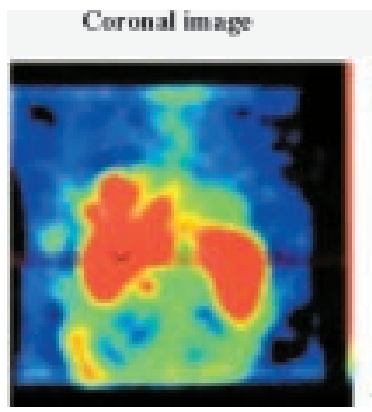

\section{Reconstructed SPECT}

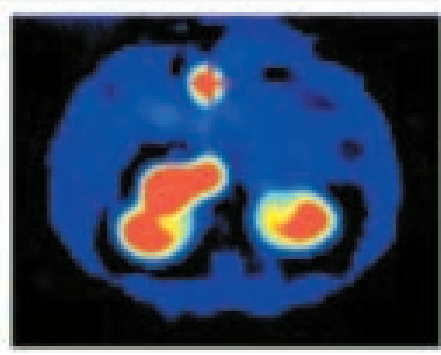

Figure 3. SPECT image obtained at 24 hours following ${ }^{90} \mathrm{Y}$-octreotide (OctreoTher) in a patient with a malignant insulinoma demonstrating a liver metastasis. of concomitant amino acid infusion. Only 4 patients who received doses $>360 \mathrm{mCi}$ without concomitant amino acid infusion developed renal toxicity; thus, all doses equal to or below $360 \mathrm{mCi}$ administered with concomitant amino acid infusion appear to have been tolerated safely ${ }^{21}$.

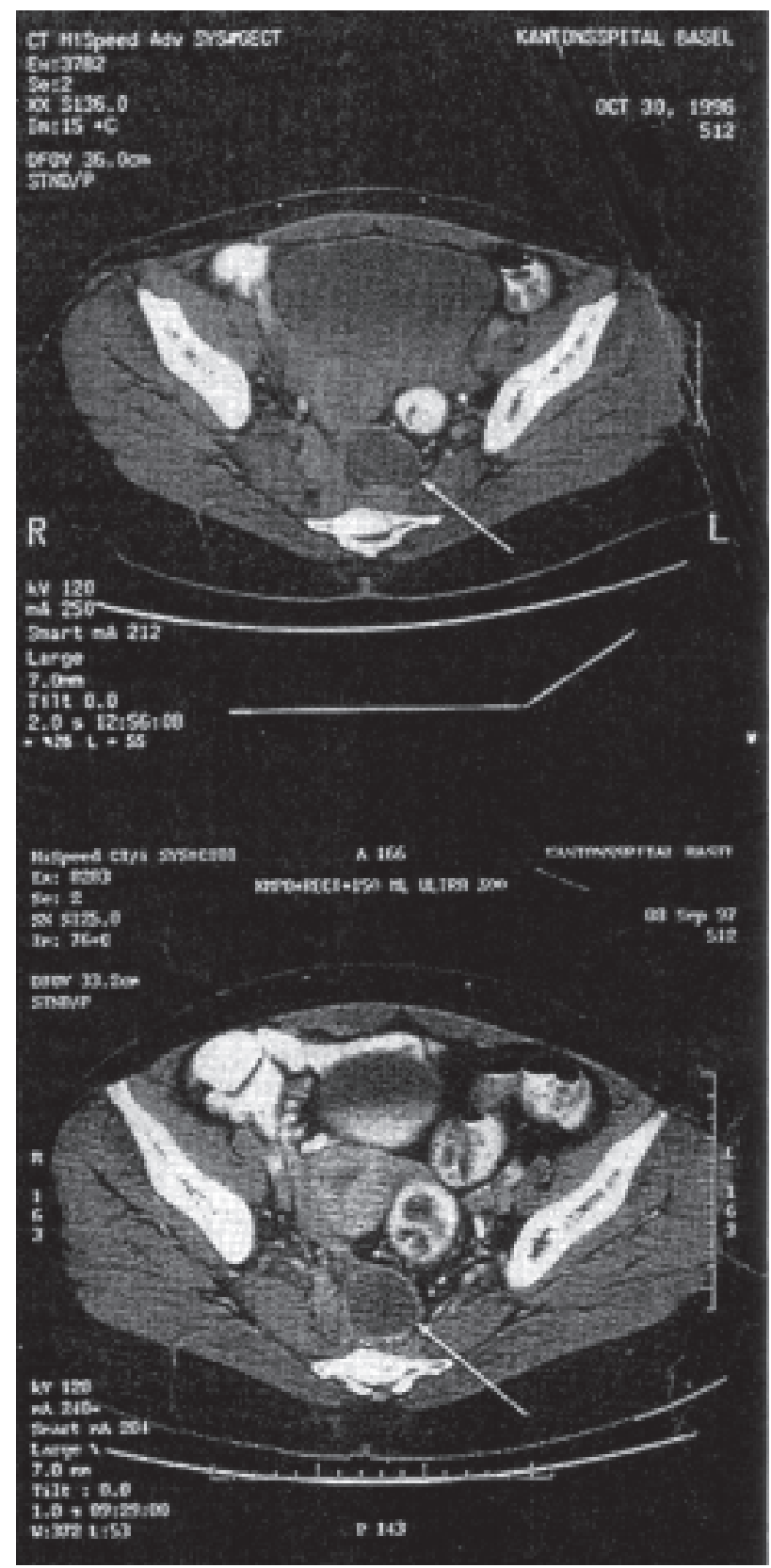

Figure 4. Contrast enhanced transaxial computed tomograph images before (top) and after treatment with [ ${ }^{90}$ Y-DOTATOC] (bottom) in the second patient. (Reproduced with permission from Lancet, 351:418,1998). 
Studies comparing the detection ability of ${ }^{123}$ IMIBG and Octreoscan have consistently shown that Octreoscan is more sensitive than ${ }^{123} \mathrm{I}-\mathrm{MIBG}$ and it will also probably locate the same lesions identified by ${ }^{131} \mathrm{I}-$ $\mathrm{MIBG}^{4,12}$. Thus, therapy with either OctreoTher or ${ }^{90} \mathrm{Y}$ DOTATOC will probably be applicable to a larger population of patients with NET than therapy with

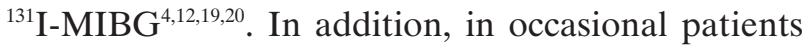
mainly with hepatic metastases, who show complementary uptake with ${ }^{123}$ I-MIBG to non-octreotide avid lesions, concomitant use of ${ }^{131} \mathrm{I}-\mathrm{MIBG}$ and OctreoTher or ${ }^{90}$ Y-DOTATOC may be another therapeutic option $^{4,12}$. As shown from data derived from patients treated with ${ }^{131} \mathrm{I}-\mathrm{MIBG}$, the main predictor of the outcome of therapy with either OctreoTher or ${ }^{90} \mathrm{Y}$-DOTATOC is principally the extent of the disease and the total tumour load ${ }^{2,15}$.

In order to overcome the limitations of administering tumouricidal doses of radiotherapy to non-octreotide avid lesions and the lack of uptake to certain areas of the tumours due to tumour heterogeneity several $\beta$-emitters, in addition to ${ }^{90} \mathrm{Y}$, such as ${ }^{153} \mathrm{Sm}$, ${ }^{177} \mathrm{Lu}$ and ${ }^{186} \mathrm{Re}$ are being considered for therapy when linked to somatostatin analogues (Table 2$)^{11}$. It has been suggested that for octreotide avid tumours radionuclear therapy with the combination of ${ }^{177} \mathrm{Lu}$ and ${ }^{90} \mathrm{Y}$ will probably be more efficacious than treatment with ${ }^{90} \mathrm{Y}$ alone. In addition, other somatostatin analogues are in development and are expected to show superior imaging and therapeutic properties compared to octreotide.

\section{FUTURE PROSPECTS}

It is likely that 'internal radiotherapy' for the treatment of NET will develop considerably within the next few years. Until now, indications with ${ }^{131} \mathrm{I}-\mathrm{MIBG}$ have been limited to palliative treatment of malignant chromaffin cell and carcinoid tumours, most often at an advanced disease stage. Therapeutic efficiency has been improved by increasing the injected activity, particularly in patients with limited tumour burden. The recent application of ${ }^{90} \mathrm{Y}$-labelled somatostatin analogues represents a major advantage as these radionuclides can be taken up by a wider number of $\mathrm{NET}^{12}$. To be fully efficient, these radionuclides should probably be injected into patients with small tumour targets (not more than $1-2 \mathrm{~cm}$ in diameter). For large macroscopic targets, internal radiotherapy is clearly inefficient be- cause of low and particularly heterogeneous tumour uptake. Although combination therapy with both ${ }^{131} \mathrm{I}$ MIBG and OctreoTher remains an attractive theoretical option for tumours that demonstrate diagnostic uptake, the only currently established solution is to perform as complete a surgical resection as possible subsequent to injection of diagnostic activity to ensure good uptake of the radiopharmaceutical by tumour targets. If injections are repeated regularly, it is likely that the disease can at least be stabilized for a period of several years if not cured. Finally, it may be hoped that the efficacy of internal radiotherapy will be improved by co-administration of chemotherapeutic drugs whose radiosensitising properties may be synergistic with that of irradiation ${ }^{2}$.

\section{REFERENCES}

1. Arnold R, Frank M, Kadjan U, 1994 Management of gastroenteropancreatic endocrine tumours: the place of somatostatin analogues. Digestion 55: 107-113.

2. Chatal JF, Le Bodic MF, Kraeber-Bodere F, Rousseau C, Resche I, 2000 Nuclear Medicine application for neuroendocrine tumours. World J Surg 24: 1285-1289.

3. Solcia E, Rindi G, Paolotti D, et al, 1999 Clinicopathological profile as a basis for classification of the endocrine tumours of the gastroenteropancreatic tract. Ann Oncol 10: S9-S15.

4. Kaltsas G, Korbonits M, Heintz E, et al, 2001 Comparison of somatostatin analogue and meta-iodobenzylguanidine (MIBG) radionuclides in the diagnosis and localisation of advanced neuroendocrine tumours. J Clin Endocrinol Metab 86:895-902.

5. Lamberts SWJ, Krenning EP, Reubi JC, 1991 The role of somatostatin and its analogues in the diagnosis and treatment of tumours. Endo Rev 12: 561-581.

6. Krenning EP, Kwekkeboom DJ, Pauwels S, Kvols LK, Reubi JC, 1995 Somatostatin receptor scintigraphy. Nucl Med Ann, New York: Raven Press 1-50.

7. Krenning EP, Kwekkeboom DJ, Bakker WH, et al, 1993 Somatostatin receptor scintigraphy with [111InDTPA D Phe1] and [123ityr3] octreotide: the Rotterdam experience with more than 1000 patients. Eur J Nucl Med 20: 716-731.

8. Krenning EP, De Jong M, Kooij PPM, et al, 1999 Radiolabelled somatostatin analogue(s) for peptide receptor scintigraphy and radionuclide therapy. Ann Oncol 10: S23S29.

9. Kaltsas GA, Mukherjee JJ, Plowman PN, Grossman AB, 2001 The role of chemotherapy in the non-surgical management of malignant neuroendocrine tumours. Clin Endocrinol 55: 575-587.

10. Borbardieri E, Maccauro M, de Deckere E, Savelli G, Chiti A, 2001 Nuclear medicine imaging of neuroendocrine tumours. Ann Oncol 12: S51-S61. 
11. Wiseman GA, Kvols LK, 1995 Therapy of neuroendocrine tumours with radiolabelled MIBG and somatostatin analogues. Semin Nucl Medicine Vol XXV 3): 272-278.

12. Kaltsas GA, Mukherjee JJ, Grossman AB, 2001 The value of radiolabelled MIBG and octreotide in the diagnosis and management of neuroendocrine tumours. Ann Oncol 12: S47-S50.

13. De Jonk M, Bakker WH, Krenning EP, et al, 1997 Yttrium-90 and Indium-111 labelling, receptor binding and biodistribution of [DOTA,D-Phe1,Tyr3] octreotide, a promising somatostatin analogue for radionuclide therapy. Eur J Nuc Med 24: 368-371.

14. Taal BG, Hoefnagel CA, Valdes Olmos RA, Boot H, 1996 Combined diagnostic imaging with 131I-MIBG and 111Inpentetreotide in carcinoid tumours. Eur J Cancer 32:19241932.

15. Mukherjee JJ, Kaltsas GA, Islam N, Plowman PN, Hikmat J, Btitton KE, Reznek RH, Jenkins PJ, Chew SL, Monson JP, Besser GM, Grossman AB, 2001 Treatment of metastatic carcinoids, phaeochromocytomas, paragangliomas and medullary thyroid carcinoma of the thyroid with 131I-metaiodobenzylguanidine. Clin Endocrinol $55: 47-60$
16. Adelstein SJ, 1993 The Auger process: A therapeutic promise? AJR 160: 707-713.

17. Smith CM, Liu J, Chen T, et al, 2000 OctreoTherTM: ongoing early clinical development of a somatostatin-receptor-targeted radionuclide antineoplastic therapy. Digestion 62:69-72.

18. Hammond PJ, Wade AF, Gwilliam ME, et al, 1993 Amino acid infusion blocks renal tubular uptake of an indium-labelled somatostatin analogue. Br J Cancer 67:14371439.

19. Paganelli P, Zoboli S, Cremonesi HR, Chinol M, 1999 Receptor mediated radionuclide therapy with $90 \mathrm{Y}-$ DOTA-D-Phe1-Tyr3-octreotide: preliminary report in cancer patients. Cancer Biother. Pharmacol. 14: 477

20. Macke HR, Behe M, Froidevaux S, et al, 1997 DOTA-DPhe 1)-Tyr 3)-octreotide (DOTATOC): a unique somatostatin receptor ligand for labelling with a variety of metalic radionuclides. J Nuc Med 38 (Suppl): 18P (Abs. 57).

21. Otte A, Mueller-Brand J, Dellas S, et al, 1998 Ytrium-90DOTA-octreotide treatment of somatostatin receptor positive tumours. Lancet 351: 417-418. 